\title{
Short-Term Wave Forecasting with AR models in Real-Time Optimal Control of Wave Energy Converters
}

\author{
Francesco Fusco and John V. Ringwood \\ Electronic Engineering Department \\ National University of Ireland Maynooth \\ Maynooth, Co. Kildare, Ireland \\ Email: francesco.fusco@eeng.nuim.ie, john.ringwood@eeng.nuim.ie
}

\begin{abstract}
Time domain control of wave energy converters requires knowledge of future incident wave elevation in order to approach conditions for optimal energy extraction. Autoregressive models revealed to be a promising approach to the prediction of future values of the wave elevation only from its past history. Results on real wave observations from different ocean locations show that AR models allow to achieve very good predictions for more than one wave period in the future if the focus is put on low frequency components, which are the most interesting from a wave energy point of view. For real-time implementation, however, the lowpass filtering introduces an error in the wave time series, as well as a delay, and AR models need to be designed so to be as robust as possible to these errors.
\end{abstract}

\section{INTRODUCTION}

Wave energy conversion in most devices is based either on relative oscillation between bodies or on oscillating pressure distributions within fixed or moving chambers. Oscillators generally have pronounced resonances, which enable efficient power absorption in certain wave conditions. In order, however, to cope with the variations of wave spectra a control system can be designed to alter the oscillator dynamics such that the efficient energy conversion occurs in a wide range of wave conditions [1].

The control approach in the early stage of wave energy, consisted of frequency domain relationships regulating the dynamics of the system to be tuned for maximum energy absorption at different peak frequencies corresponding to different incoming wave spectra [1],[2]. Although being an advantageous approach in real sea spectra, it does not generally allow control on a wave by wave basis, that is a real-time control that could significantly raise the device productivity and therefore its economical viability. Real-time optimal control can be directly derived from the aforementioned optimal frequency relationships, and its main difficulties raise from the fact that their transformation in the time domain results in non causal transfer functions, so that the conditions for optimal power absorption can be realised only if future motion of the device, or future incident wave, are known [1],[2],[3].

As an example, Fig. 1 represents a possible control scheme for a generic oscillating body, where the controlled variable is its oscillation velocity, $u$, and the control action is performed through a force $F_{c}$. The optimal reference velocity, $\hat{u}_{d}$, is computed by an algorithm that requires future values of the incident wave elevation $\eta$, that must be provided by a predictor, which in this case is based only on the past history of the signal itself. Depending on the specific device operating principle, as well as on the general control strategy adopted and on the available instrumentation, the involved quantities may vary, but the logic can always be described by a structure as the one proposed in Fig. 1, where a certain physical quantity needs to be predicted in order for the reference to be as close as possible to the optimality condition. This quantity may be the wave excitation force or the oscillation velocity for an oscillating body, the dynamic air pressure inside the chamber for an oscillating water column, the overtopping water for an overtopping device, and all of them are ultimately dependent on the incident wave elevation.

The approach to prediction from only the past history of the signal itself presents several advantages with respect to the reconstruction of the wave field from distant observations, which requires an array of measurements (to deal with multidirectionality and to separate incident from refracted waves) and complex prediction models, thus resulting in increased instrumentation costs. Linear Autoregressive (AR) models have been proposed as an efficient and effective solution for the prediction of the oscillation velocity [4] and the wave elevation [5],[6],[7]. Particularly in [6] and [7], AR models were shown to outperform even neural networks, with very accurate predictions obtained for more than one period on real wave elevation time series, when the high frequency components were filtered out.

In this paper, the identification and estimation of AR models for short term wave forecasting is presented in section II, together with a methodology for the characterisation of the probability distribution of the predictions through confidence intervals. More exhaustive results on real observations from different ocean locations and in a variety of climates are presented in section III. A real-time implementation of this prediction procedure, however, requires the design of a realisable lowpass filter (so far, only ideal off-line lowpass filters were applied). This issue and the consequences on the AR 


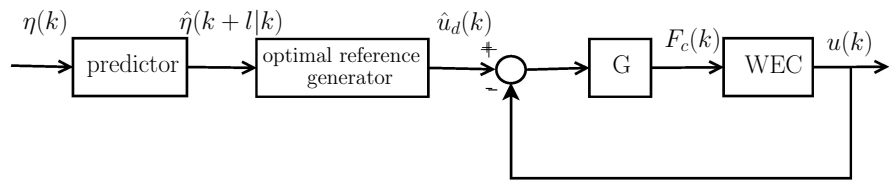

Fig. 1. Time domain control of wave energy converters requires future knowledge of the incident wave elevation to approach optimality

model are discussed in section IV.

\section{Autoregressive MOdel}

The wave elevation $\eta(k)$ is supposed to be linearly dependent on a number $n$ of its past values:

$$
\eta(k)=\sum_{i=1}^{n} a_{i} \eta(k-i)+\zeta(k)
$$

where a disturbance term $\zeta(k)$ has been also included. If the parameters $a_{i}$ are estimated and the noise is supposed to be Gaussian and white, the best $l$-step ahead prediction of the future wave elevation at instant $k, \hat{\eta}(k+l \mid k)$, is given by:

$$
\hat{\eta}(k+l \mid k)=\sum_{i=1}^{n} \hat{a}_{i}(k) \hat{\eta}(k+l-i \mid k)
$$

where, obviously, $\hat{\eta}(k+l-i \mid k) \equiv \eta(k)$ if $k+l-i \leq k$ (i.e. information already acquired and no need of prediction).

The general shape of the prediction function is completely determined by the poles, $p_{i}$, of the corresponding transfer function, and its particular realisation is determined, at each sampling instant, by the past values of the time series [8]. It is particularly interesting to analyse the shape of the forecasting function (2) in the case of $m / 2$ (when $m$ is even) couples of complex-conjugate poles $p_{i}$ and $p_{i}^{*}$ :

$$
\hat{\eta}(k+l \mid k)=\sum_{i=1}^{m / 2} c_{i}(k)\left|p_{i}\right|^{l} \sin \left(\angle p_{i} k+\varphi_{i}(k)\right)
$$

Thus, an AR model with only complex-conjugate poles is implicitly a cyclical model, where the frequencies are related to the phase, $\angle p_{i}$, of each pole and the amplitudes and phases of the harmonic components are related to the last $n$ measurements of each time instant $k$, so that they adapt to the observations [7].

\section{A. Estimation}

The AR coefficients can be estimated from a number $N$ of batch wave elevation observations. The standard approach, under the assumption of Gaussian error, is to minimise the sum of squares of the one-step ahead prediction error, through regular least squares. Because the model will be utilised for multi-steps ahead forecasting, however, either the estimation and the identification problems turn out to be ill-posed, since many models can be found which are almost equivalent in terms of one-step ahead prediction, but fare quite differently in longer-term prediction [9]. A multi-steps ahead cost functional is therefore applied, referred to as long-range predictive identification (LPRI) [10]:

$$
J_{L P R I}=\sum_{k=1}^{N} \sum_{j=N_{1}}^{N_{2}}[\eta(k)-\hat{\eta}(k \mid k-j)]^{2}
$$

This functional can be minimised using a standard algorithm for solving nonlinear least-squares problems, such as the Gauss-Newton approach, initialised with the results from regular least squares [10]. In our specific case we set $N_{1}=1$ and $N_{2}$ as the maximum prediction horizon $\left(N_{2}=50\right.$ for the Galway Bay data and $N_{2}=25$ for the Pico Island data), because we want to optimise the prediction accuracy at any lead time.

Note that, from (3), the frequencies are related to the poles, so if the parameters are kept constant, the frequencies are constant as well. An adaptivity mechanism based on the LPRI cost function (4) could then be considered, which is also proposed in [10]. However, as it will be shown in the results, section III, a static AR model keeps its validity for long time after being estimated (more than 2 hours), in spite of wave spectral variations. Adaptive AR models do not represent, therefore, a big issue at the moment and are not considered in this paper.

\section{B. Identification}

An indication about the appropriate order $n$ of the AR model to predict the wave elevation, can be obtained through minimisation of the classical criteria $A I C$, proposed by Akaike [11], and $B I C$, proposed by Schwarz [12] :

$$
\begin{aligned}
& A I C=\log \left(\hat{\sigma}^{2}\right)+r \frac{2}{N} \\
& B I C=\log \left(\hat{\sigma}^{2}\right)+r \frac{\log (N)}{N}
\end{aligned}
$$

Here, $r=n+1$ is the number of parameters to be estimated, $\hat{\sigma}^{2}$ is the value of the minimised functional (4), and not, as normally utilised, the one-step ahead error variance, a modification which is coherent with the estimation criterion and with the use of the AR model for multi-step ahead prediction purposes.

\section{Confidence intervals}

The predictions alone, as given by (2), do not give a complete enough information about the future of the wave elevation, because they are inevitably affected by an estimation error, so that it would be fundamental to have an indication about the entity of this error and about how reliable the predictions are.

If the $l$-steps ahead prediction error is Gaussian:

$$
\hat{e}(k+l \mid k)=\eta(k+l)-\hat{\eta}(k+l \mid k) \sim \aleph\left(0, \sigma_{l}^{2}\right)
$$

then the variance, $\sigma_{l}^{2}$, is all we need in order to define its probability distribution. Moreover, we can assume a confidence interval where the error is contained with a probability $\alpha$ :

$$
-p_{\frac{\alpha}{2}} \leq \hat{e}(k+l \mid k) \leq+p_{\frac{\alpha}{2}}
$$


Here, $p_{\frac{\alpha}{2}}$ is the value of the probability distribution such that:

$$
P\left\{-p_{\frac{\alpha}{2}} \leq \hat{e}(k+l \mid k) \leq+p_{\frac{\alpha}{2}}\right\}=\int_{-p_{\frac{\alpha}{2}}}^{+p_{\frac{\alpha}{2}}} p(y) d y=\alpha
$$

where $p(\cdot)$ is a Gaussian probability density function.

The estimate of the variance $\sigma_{l}^{2}$ could be calculated from the specific model parameters and from the statistics of its estimation algorithm [8]. The problem with this approach is that the confidence intervals can result to be misleading if the model is not perfectly appropriate and if its accuracy changes accordingly with the wave conditions. A more straightforward alternative is adopted at this stage, where the estimate of the variance of the forecasting error is based on the past history of the predictions:

$$
\hat{\sigma}_{l}^{2}=\frac{1}{N-1} \sum_{k=1}^{N}\left[\hat{e}(k+l \mid k)^{2}\right]
$$

where $N$ is the number of past observations available. This estimate can also be recursively updated as soon as new observations become available [13].

\section{RESULTS WITH IDEAL LOWPASS FILTERS}

The wave forecasting AR models are tested against real wave observations from different sea locations:

1) The Irish Marine Institute provided real observations from a data buoy located in Galway Bay, on the West Coast of Ireland, at approximately $53^{\circ} 130^{\prime} N$, $9^{\circ} 180^{\prime} W$ (water depth nearly $20 \mathrm{~m}$ ). Data consist of 20 minute records sets for each hour, collected at a sampling frequency of $2.56 \mathrm{~Hz}$.

2) Wave elevation time series are also available from the Atlantic Ocean at the Pico Island, in the Azores archipelago, at approximately $38^{\circ} 330^{\prime} \mathrm{N}, 28^{\circ} 340^{\prime} \mathrm{W}$ (water depth around $40 \mathrm{~m}$ ). The Pico data are collected in the form of two contiguous 30 minute records sets for each hour, with a sampling frequency of $1.28 \mathrm{~Hz}$ [14],[15],[16].

Some significant data sets, reflecting different wave climates, were picked up from all the ones available, and their spectrum is shown in Fig. 2. In particular one wide banded and one narrow banded sea state from the two sites is considered. Then, a situation where wind waves predominate is picked up from the Galway Bay data and a very high energy wave system, where the sea bottom slightly affects the wave symmetry (this was analysed through higher order spectral analysis and skewness and kurtosis indices [6]), is chosen from the Pico Island data.

The accuracy of some AR models on the selected wave time series is measured, for any lead time $l$, through the following index of fitness:

$$
\mathcal{F}(l)=\left(1-\frac{\sqrt{\sum_{k}[\eta(k+l)-\hat{\eta}(k+l \mid k)]^{2}}}{\sqrt{\sum_{k} \eta(k)^{2}}}\right) \cdot 100 \%
$$
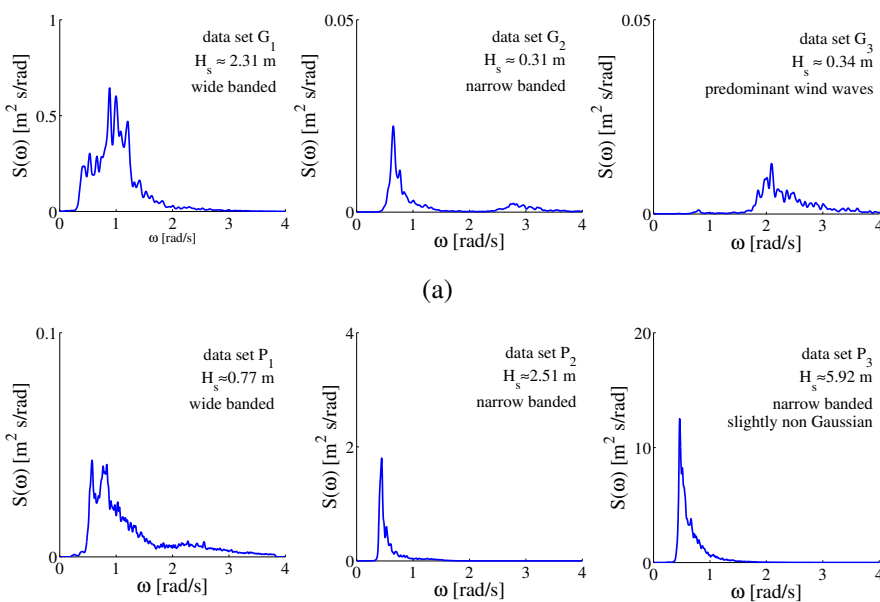

(b)

Fig. 2. Spectrum of some significant data sets: (a) Galway bay; (b) Pico island. $H_{s}$ is the significant wave height.

Here $\eta(k+l)$ is the wave elevation and $\hat{\eta}(k+l \mid k)$ is its prediction based on the information up to instant $k$. A $100 \%$ value for $\mathcal{F}(l)$ means that the wave elevation time series is perfectly predicted $l$ steps into the future. Note that the quantity $\mathcal{F}$ has a direct correspondence with the variance of the prediction error, that, as discussed in section II-C, is utilised to characterise the forecasts confidence intervals (the gaussianity of the multi-steps ahead prediction error distribution has been verified through analysis of skewness and kurtosis).

Focus has been put on the low frequency components, as they are the more interesting from a wave energy point of view, and this allows for a significant improvement of the prediction, as shown through a predictability analysis in [6],[7]. The cutoff frequency $\omega_{c}$ has been chosen accordingly to the spectral distribution of the specific sea state.

Fig. 3 shows the very good accuracy achievable with AR models when narrow banded sea states occur, with $\mathcal{F}(l)>$ $80 \%$ for predictions more than $15 s$ in the future. In the case of wide banded sea states, $G_{1}$ and $P_{1}$, the accuracy is still $\mathcal{F}(l)>80 \%$ for prediction horizons of about $10-12 \mathrm{~s}$. The sea state $G_{3}$, dominated by wind waves and affected by strong non linearities (detected through the Bispectrum [6]), gives more problems and neural networks gave better results in this case [6],[7].

It is interesting to note how the poles of the $A R$ models are estimated very close to the unit circle of the complex plane, and the corresponding frequencies map the training wave spectrum, as shown in Fig. 4.

For the sake of completeness, a screenshot comparing the 25 -steps ahead prediction from an AR model and the real wave, together with the $90 \%$ confidence interval, is plotted in Fig. 5.

\section{EFFECTS OF REAL TIME FILTERS}

In order to improve the prediction accuracy and the forecasting horizon a lowpass filtering of the wave elevation is performed on the original signal. This procedure is reasonable in view of the fact that wave energy devices, and therefore 


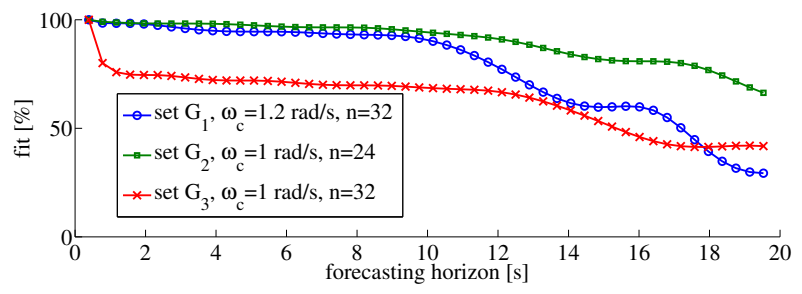

(a)

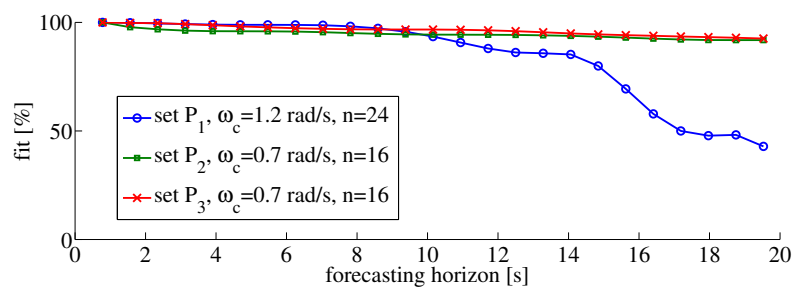

(b)

Fig. 3. Performance: (a) Galway bay; (b) Pico island.



(a)

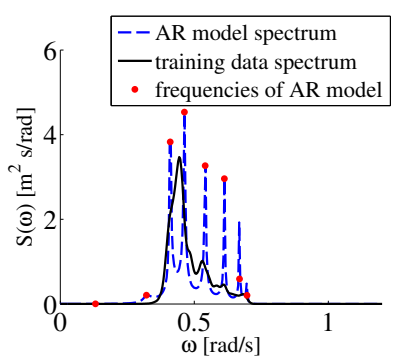

(b)
Fig. 4. Poles and frequency response of an AR model trained on a wave elevations data set.

their control algorithms, are to be optimised at the waves that contain the most energy, and they are the low frequency ones [6]. Lowpass filtering is also reasonable because what is really required to be predicted, in the control of wave energy converters, may be one of the consequences of the incident wave elevation on the device, such as the wave excitation force, and they are all filtered by the lowpass dynamics of the device itself.

AR models achieved great performance in predicting the

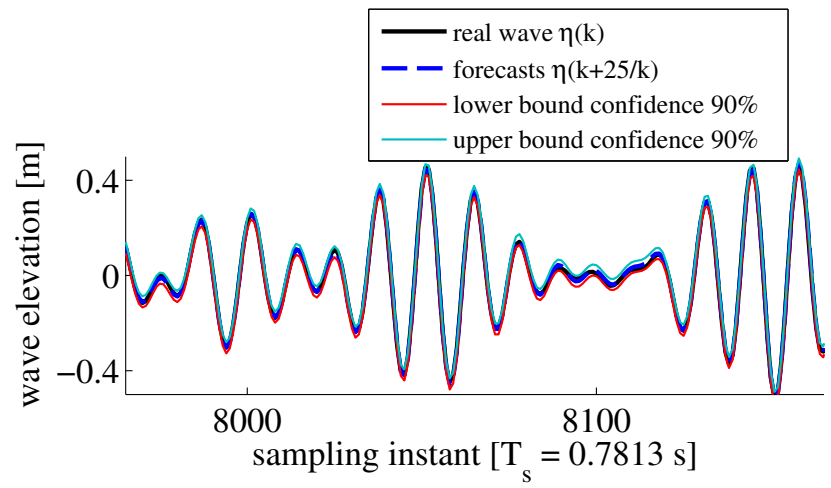

Fig. 5. Confidence interval and 25-steps-ahead predictions of an AR model with order $n=24$ on the data set $P_{2}$, filtered with cut-off frequency $\omega_{c}=$ $0.7 \mathrm{rad} / \mathrm{s}$. low frequencies, as shown in section III and in [6],[7]. In real time applications, however, lowpass filtering inevitably introduces an error, in terms of signal amplitude and delay and its effects on the forecasts need to be addressed.

\section{A. Digital filters: error and delay}

If the observed time series representing the wave elevation is $\eta^{o}(k)$, then the prediction will be focused on the filtered wave:

$$
\eta(k) \triangleq F\left(e^{j \omega}\right) \eta^{o}(k)
$$

where $F\left(e^{j \omega}\right)$ is a digital filter.

Ideally, $F\left(e^{j \omega}\right)$ would have a perfectly zero phase response and a piecewise constant amplitude response being 1 in the pass band and 0 in the stop band. This, however, leads to a non causal impulse response and, therefore, to a filter non computationally realisable in real time [17]. Inevitably, a realisable digital filter will introduce two inconvenient issues:

1) A non zero phase response, which in the time domain means a delay. In the best of the hypothesis this delay is a constant, if the phase response is linear in the frequency, otherwise different frequency components of the wave elevation will be delayed by a different time. In any case it is important that the delay is very small, because any sample of delay introduced by the filter is a sample lost in the achievable prediction horizon.

2) A transition band in the amplitude response, which causes frequency components in the stop band being not completely removed by the filter and components in the pass band being attenuated.

The two traditional approaches to digital filters design are the discretisation of an analogue filter, which generates an Infinite Impulse Response (IIR) filter, or the direct synthesis of a Finite Impulse Response (FIR) discrete time filter (frequency response with only zeros). The big advantage of FIR filters is the possibility to obtain a perfect linear phase response and so a constant delay. IIR filters, on the other hand, allow to obtain far better amplitude responses with very low orders, although they have a non-linear phase response.

Several FIR and IIR filters were designed and their performance was quantified through:

- The delay $\tau$, which is perfectly known for FIR filters. In the case of IIR filters it is identified by matching the output of the filter with the ideal filtered signal.

- The Mean Error, $M E$, between the filtered time series, $\eta(k)$, and the correspondent ideally filtered time series, $\eta^{i}(k)$, normalised with respect to the standard deviation of the ideally filtered time series:

$$
M E=\frac{1}{N-\tau-t_{r}+1} \frac{\sum_{k=t_{r}}^{N-\tau}\left|\eta(k+\tau)-\eta^{i}(k)\right|}{\frac{1}{N} \sqrt{\sum_{k=1}^{N} \eta^{i}(k)^{2}}}
$$

Here $N$ is the length of the filtered signal, $\tau$ is the estimated delay (or exact for a FIR filter) and $t_{r}$ is the transient time (in samples), which in theory is infinitely 


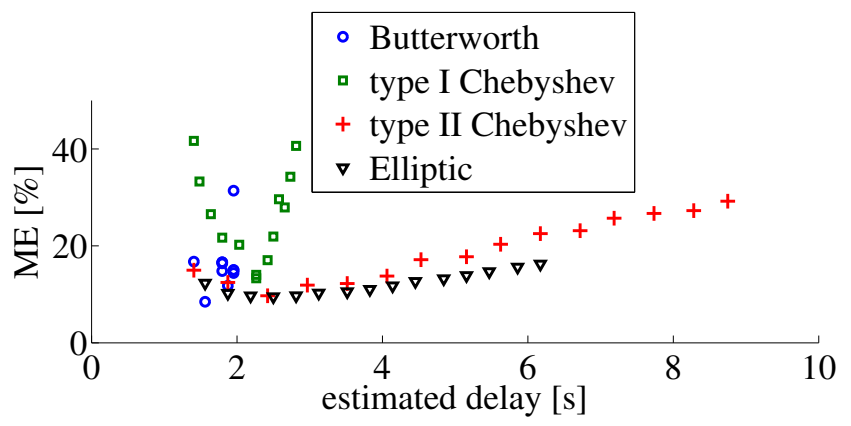

(a)

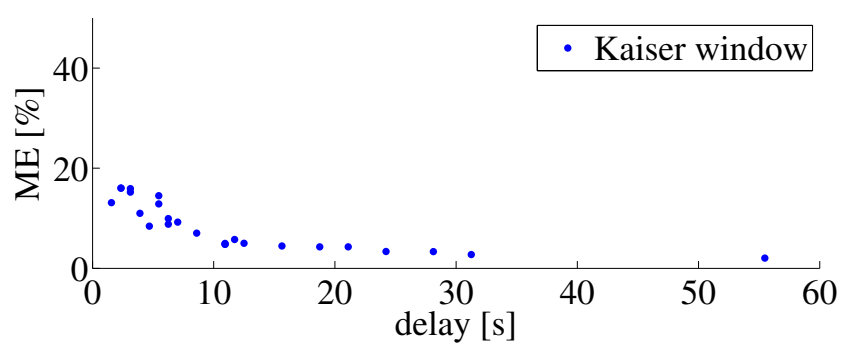

(b)

Fig. 6. Performance of digital filters on $P_{2}$ data set: $(a)$ IIR filters; $(b)$ FIR filters.

long for IIR filters, but that tends towards zero if they are stable so it can be negligible after a certain point.

Figure 6 shows the distribution of a number of digital filters, with cutoff frequency $\omega_{c}=1 \mathrm{rad} / \mathrm{s}$, with respect to $\tau$ and percentage $M E$, tested against the wave elevation data set from Pico island, $P_{2}$ in Fig. 2. The FIR filters were designed through Kaiser windows with different specifications for the transition band and the filter's order [17], while the IIR filters are discretisation, through, the bilinear transform of classical analogue filters. In particular, from figure 6(a), the best IIR filters seem to be low order Elliptic, Butterworth and type I Chebyshev filters. The higher the order, in fact, the stronger the non-linearity of the phase, so that the phase distortion raises the error, in spite of a sharper transition of the amplitude response. In the case of FIR filters, figure 6(b), the $M E$ decreases with increasing order (directly proportional to the delay) because of the perfectly linear phase. The delay, however, quickly gets very high, so that only very small order FIR filters can be acceptable.

In any case, it seems hard to obtain a $M E<10 \%$ with a delay $\tau<5 s$ so that it is important for the prediction algorithm, in this case Autoregressive models, to be robust enough in response to errors of this order of magnitude. Of course, deeper research may be led in the direction of finding an optimal filter minimising both the delay and the $M E$, but the focus at the moment is put on the ability of wave forecasting AR models to properly deal with such errors in the observations time series.

\section{B. AR model wave forecasts sensitivity}

The sensitivity of the forecasts of an AR model to errors in the observations is the same as the sensitivity to its initial conditions. From (2), in fact, the predictions are computed from the free evolution of the model based on the last $n$ measurements, where $n$ is the order. In order to analyse the sensitivity, it is handy to write the state space form of the AR predictor, where the state vector is defined as:

$$
\begin{aligned}
& x^{k}(j) \triangleq[\hat{\eta}(k+j \mid k) \quad \hat{\eta}(k+j-1 \mid k) \quad \ldots \\
& \ldots \hat{\eta}(k+j+1-n \mid k)]^{T}
\end{aligned}
$$

Here, $\hat{\eta}(k+j \mid k)$ is the $j$-steps ahead prediction of the wave elevation based on the information up to instant $k$ and $\hat{\eta}(k+$ $j / k)=\eta(k+j)$ if $k+j \leq k$, as the information is already acquired.

At any instant $k$, the evolution of the state vector $x^{k}(j)$ represents the evolution of the $j$-steps ahead wave prediction starting from the initial state $x^{k}(0)$ and it is regulated by the following iterative form:

$$
\begin{aligned}
x^{k}(j+1) & =A x^{k}(j) \\
\hat{\eta}(k+j \mid k) & =C x^{k}(j)
\end{aligned}
$$

where

$$
\begin{aligned}
A & =\left[\begin{array}{cccc}
a_{1} & a_{2} & \ldots & a_{n} \\
1 & 0 & \ldots & 0 \\
\ldots & \ddots & \ldots & \vdots \\
0 & \ldots & 1 & 0
\end{array}\right] \\
C & =\left[\begin{array}{llll}
1 & 0 & \ldots & 0
\end{array}\right]
\end{aligned}
$$

It follows that the direct relationship between the initial state $x^{k}(0)$ and the $j$-steps ahead prediction is:

$$
\hat{\eta}(k+j \mid k)=C A^{j} x^{k}(0)
$$

where the initial state contains the last $n$ observations available at instant $k$ :

$$
x^{k}(0)=\left[\begin{array}{lll}
\eta(k) & \eta(k-1) \quad \ldots \eta(k+1-n) \ldots
\end{array}\right]^{T}
$$

An error in the wave observations $\eta(k)$, such as the one introduced by a real time digital filter, means an error in the initial state $x^{k}(0)$, named $\Delta x^{k}(0)$, which will have some additive effect $\Delta \hat{\eta}(k+j \mid k)$ on the $j$-steps ahead prediction:

$$
\hat{\eta}(k+j \mid k)+\Delta \hat{\eta}(k+j \mid k)=C A^{j}\left[x^{k}(0)+\Delta x^{k}(0)\right]
$$

By exploiting some results about linear algebra, it can be shown that, for any error $\Delta x^{k}(0)$ and for any norm operator $\|\cdot\|$ :

$$
\underline{\sigma}\left(C A^{j}\right) \leq \frac{\|\Delta \hat{\eta}(j+k \mid k)\|}{\left\|\Delta x^{k}(0)\right\|} \leq \bar{\sigma}\left(C A^{j}\right)
$$

where $\underline{\sigma}(\cdot)$ and $\bar{\sigma}(\cdot)$ are respectively the minimum and the maximum singular value operators.

In our specific case $C A^{j} \in \mathbb{R}^{1 \times n}$ so that only 1 singular value is non zero and will be considered as a measure of sensitivity. In Fig. 7 the singular value of $C A^{j}$ is shown, in $d B$, where the matrix $A$ corresponds to an AR model of order $n=16$, trained on the wave elevation data set $P_{2}$, as from figure 2(b). The sensitivity is clearly very high and gets worse 


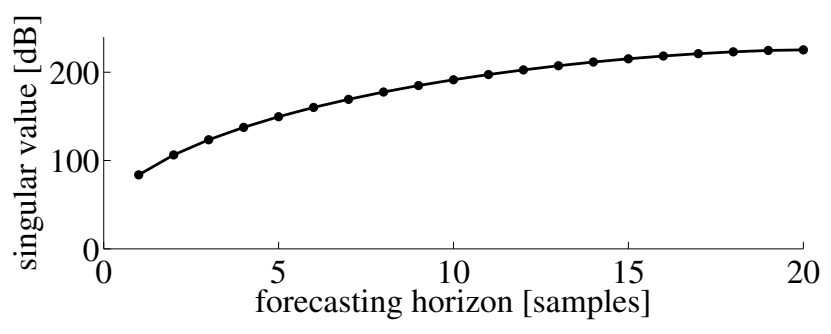

Fig. 7. Maximum singular value, in $\mathrm{dB}$, of the matrix $C A^{j}$

and worse while the forecasting horizon increases, as the error accumulates:

$$
\begin{aligned}
\|\Delta \hat{\eta}(k+1 \mid k)\| & \leq 1.5402 \times 10^{4}\left\|\Delta x^{k}(0)\right\| \\
\|\Delta \hat{\eta}(k+5 \mid k)\| & \leq 3.0381 \times 10^{7}\left\|\Delta x^{k}(0)\right\| \\
\|\Delta \hat{\eta}(k+10 \mid k)\| & \leq 3.7629 \times 10^{9}\left\|\Delta x^{k}(0)\right\|
\end{aligned}
$$

A very small error in the observations has a huge effect on the prediction and in fact this AR model heavily diverges if a very small noise is added to the observations. This is a serious issue to deal with and further work should be focused on the design of robust AR models, which are able to reject certain kind of disturbances, in particular the ones introduced by a real time filter, while still maintaining a good accuracy in the wave predictions.

\section{CONCLUSION}

This study was focused on the problem of short term wave prediction, which is a central topic in the wave energy field, in order to allow a better effectiveness and economic viability of any WEC. An analysis of AR models, in section II, highlighted how they implicitly represent cyclical models where the frequencies are easily estimated with linear least squares (as they are related to the regression coefficients). The amplitudes and phases of each harmonic component is, moreover, implicitly adaptive to the recent observations due to the regression terms of the model.

Results on real wave elevation data from the Galway Bay and from the Pico Island, in section III, showed how an AR model estimated on a batch training data set, offered very good accuracy up to 15 seconds (in some cases even 20 seconds) predictions for the low frequency components of the waves. It was shown also how the frequencies are automatically estimated in the significant range of the wave spectrum of the training set. The possibility to adapt the model in order to track variations of the wave spectrum through variable frequencies in the model, was also analysed. Because, however, static AR models were shown to maintain their prediction ability for long times (no performance decrease for 2 hours simulations), their adaptivity is not seen as a main issue at the moment, and also a simple periodic batch estimate may be a feasible solution, or the use of a set of AR models, estimated from different sea conditions, and a switching logic deciding which one is the more appropriate in real time.

In real-time applications, however, the lowpass filtering introduces an error in the wave elevation time series and a delay. A preliminary analysis of the sensitivity of the estimated AR models, in section IV-B, showed how they can diverge for very small deviations of the initial conditions, and therefore of the observations. The issue of designing a more robust AR models was highlighted and further work needs therefore to be done in this direction, in order to make this promising wave forecasting approach ready for use in a time domain control framework for wave energy converters.

\section{ACKNOWLEDGMENT}

The authors would like to thank the Irish Marine Institute for providing the data from the Galway Bay. The data from Pico Island is available by courtesy of the Centre of Climate, Meteorology and Global Change of the University of the Azores, projects CLIMAAT; CLIMARCOST; MacSIMAR (FEDERPIC_Interreg_IIIB \& MAC; MAC2.3/A3; 03/MAC/2.3/A5; 05/MAC/2.3/A1; MAC/1/AO89). The research was funded by the Irish Research Council of Science, Engineering and Technologies (IRCSET) under the Embark Initiative.

\section{REFERENCES}

[1] U. A. Korde, "Control system applications in wave energy conversion," Proceedings of the OCEANS 2000 MTS/IEEE Conference and Exhibition, vol. 3, pp. 1817-24, 2000.

[2] J. Falnes, Ocean Waves and Oscillating Systems. Cambridge University Press, 2002

[3] - "A review of wave-energy extraction," Marine Structures, vol. 20, pp. 285-201, 2007.

[4] U. A. Korde, "Efficient primary energy conversion in irregular waves," Ocean Engineering, vol. 26, pp. 625-651, 1999.

[5] M. P. Schoen, J. Hals, and T. Moan, "Wave prediction and fuzzy logic control of wave energy converters in irregular waves," Proceedings of the 16th Mediterranean Conference on Control and Automation, Ajaccio, France, pp. 767-772, 2008.

[6] F. Fusco and J. Ringwood, "A study on short-term sea profile prediction for wave energy applications," Proc. of the 8th European Wave and Tidal Energy Conference (EWTEC), pp. 756-765, Uppsala, Sweden, 2009.

[7] — , "Short-Term Wave Forecasting for Real-Time Control of Wave Energy Converters," accepted for publication on IEEE Trans. on Sustainable Energy, 2010.

[8] G. E. P. Box, G. M. Jenkins, and G. C. Reinsel, Time Series Analysis: Forecasting and Control. Prentice-Hall, 1994.

[9] L. Piroddi and W. Spinelli, "Long-range nonlinear prediction: A case study," Proceedings of the 42nd IEEE Conference on Decision and Control, Maui, Hawaii USA, pp. 3984-3989, 2003.

[10] D. S. Shook, C. Mohtadi, and S. L. Shah, "Identification for longrange predictive control," IEEE Proceedings D (Control Theory and Applications), vol. 138, no. 1, pp. 75-84, 1991.

[11] H. Akaike, "A new look at the statistical model identification," IEEE Transactions on Automatic Control, vol. 19, pp. 716-723, 1974.

[12] G. Schwarz, "Estimating the dimension of a model," The Annals of Statistics, vol. 6, no. 2, pp. 461-464, 1978.

[13] R. Isermann, Fault-Diganosis Systems. An Introduction from Fault Detection to Fault Tolerance. Springer, 2006.

[14] R. Esteves, A. Valente, M. Costa, F. Reis, and E. Azevedo, "Dados direccionais de agitao martima nos aores (projecto climaat-clima e meteorologia dos arquiplagos atlnticos)," 6ts Jornadas Portuguesas de Engenharia Costeira e Porturia, PIANC, Funchal, 2009.

[15] E. Azevedo and M. Rodrigues, "Projectos climaat \& climarcost clima e meteorologia dos arquiplagos atlnticos, clima martimo e costeiro," Workshop Intenacional sobre Clima e Recursos Naturais nos Pases de Lngua Portuguesa, WSCRA08, 2008.

[16] C. Barrera, E. Azevedo, M. Rueda, M. Gelado, and L. O., "Real-time monitoring network in the macaronesian region as a contribution to the coastal ocean observations panel (COOP)," Journal of Operational Oceanography, vol. 1, pp. 59-69, 2008.

[17] A. V. Oppenheim and R. W. Schafer, Discrete-Time Signal Processing. Prentice Hall International Inc., 1989. 